\title{
EFFECTS OF RFI ON SOLAR MICROWAVE BURSTS OBSERVED WITH HIGHTIME RESOLUTION
}

\author{
LUO XIANHAN \\ Department of Geophysics \\ Peking University \\ Beijing, CHINA
}

\begin{abstract}
Solar microwave burst observations with high time resolution $(\sim 1 \mathrm{~ms})$ are important but difficult to make. It is shown by the experiments of radiometer at wavelength $21 \mathrm{~cm}$ on $1 \mathrm{~ms}$ time scale that some ultrafast time structures in microwaves, which includes spike impulses, switch-on and switch-off structures, etc., may not be from solar emission but from RFI (radio-frequency interference) or from radiometer itself. Because of the uncertainty at $21 \mathrm{~cm}$ and other several wavelengths, we suggest that joint observations of the solar microwave bursts on $1 \mathrm{~ms}$ time scale should be carried out on the peak years of the 22nd solar activity cycle.
\end{abstract}

\section{INTRODUCTION}

Recently, some observations of solar microwave bursts have been made with high time resolutions up to 1 millisecond (for example Slottje 1978 (1); Kaufmann 1978 (2); Kaufmann et al. 1980 (3)). These observations revealed new aspects of solar activity and have much significance for studying radio physics of the Sun. In this paper, we shall describe the progress of the observations with high time resolutions in China, and present some problems and proposals.

\section{SITUATIONS OF THE OBSERVATIONS IN CHINA}

In May 1981, scientists at Beijing Observatory of China first observed some ultra-fast fine structures of the microwave bursts at the frequency of $2.84 \mathrm{GHz}$ $(10.16 \mathrm{~cm})(4)$, and obtained some interesting results. Then Peking University, Yunnan Observatory, Beijing Normal University and Nanjing University et al. also made similar observations and experiments at different wavelengths (see Table 1).

The millisecond recording equipments usually were reassembled on the basis of solar radio telescopes with second scale record (5). In the early 1980s, because the storage of the microcomputer was small, the observations with millisecond scale could only last for several minutes. Not until July 1986, could continuously fast recording observations of Yunnan Observatory with $10 \mathrm{~ms}$ scale at $21 \mathrm{~cm}$ last for several hours (6). 
For the study of the solar radio fast time structures at different frequencies, in January 1987 the three band synchronous observations of 8.2, 10.6 and $21 \mathrm{~cm}$ with $1 \mathrm{~ms}$ scale were expanded at Yunnan Observatory (7).

\section{TABLE I OBSERVATIONS OF ULTRA FAST MICROWAVE BURSTS}

\begin{tabular}{|c|c|c|c|c|}
\hline (cm) & $(\mathrm{MHz})$ & $\begin{array}{c}\text { Time } \\
\text { Resolution } \\
\text { (ms) }\end{array}$ & $\begin{array}{c}\text { Observation } \\
\text { Date }\end{array}$ & Institution \\
\hline$-\infty$ & ----- & $-\infty--$ & $-----n----\infty--1$ & 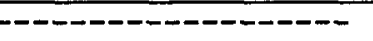 \\
\hline 10.6 & 2840 & 1 & April, 1981 & Beijing observatory \\
\hline 21 & 1420 & 1 & May-Aug. , 1983 & $\begin{array}{l}\text { Peking University, } \\
\text { Beijing Observatory }\end{array}$ \\
\hline 10.3 & 2902 & 1 & August, 1983 & Yunnan Observatory \\
\hline 2 & 15000 & 1 & 1984 & Beijing Normal Univ \\
\hline 3.2 & 9375 & 1 & 1984 & Nanjing University \\
\hline 21 & 1420 & 10 & July, 1986 & Yunnan Observatory \\
\hline 8.2 & 3670 & & & $-\infty-$ \\
\hline 10.6 & 2840 & 1 & January, 1987 & Yunnan Observatory \\
\hline 21 & 1420 & & & \\
\hline
\end{tabular}

\section{EXPERIMENTS OF RADIOMETER AT WAVELENGTH 21 CM}

\section{(1) Spike Impulses and Power Supply Interferences}

Fig. 1 shows the fast time structures recorded by the $21 \mathrm{~cm}$ radio telescope toward the Sun at Peking University on August 7, 1983. The A,B,C,D and E of Fig. 1(a) were enlarged in Fig. 1(b). We are not sure that these are really the solar signals, because at the same time on second scale recorder we did not receive the radio bursts.

On August 24, 1983, we used an absorbing load to replace the antenna, and did some experiments. When the power supply of an electric fan was switched off, we obtained some data much similar to the fast time structures (Fig. 2).

Comparing Fig. 1 and Fig. 2, we found the magnitudes, widths and fine structures of A,B in Fig. 1(b) are quite similar with the K, L in Fig. 2(b). These may be the indications that some spike impulse recorded during the antenna towards the Sun might have come from RFI, rather than from the solar emission. 


\section{(2) On the Stability of the System}

On August 5, 1983, during the antenna towards the Sun, we obtained the data and recorded on magnetic tape for several seconds. The data were processed on the same day and converted into pictures as shown in Fig. 3(a). However, from that day forward, the figures were changing about every time, whenever we converted them into pictures with the same methods. For example, it was shown respectively in (b), (c) and (d) of Fig. 3.

Comparing these figures, we found that some man-made "switch-on and switch-off structures" lasted for $250 \mathrm{~ms}$; the magnitudes and numbers of some "spike impulses" also changed; in addition to this, there were some fluctuations in part of the background level of Fig. 3(d).

Above-mentioned changes of Fig. 3 were formed by the system instability (jumps and fluctuations of gain, etc.) under weak signals. They are related to some radio interferences and instrumental quality, and certainly no relation at all to the solar radio bursts.

\section{DISCUSSIONS AND SUGGESTIONS}

Besides the above effects of radio interference during observations and experiments, there were effects of other radio interference at several wavelengths (for example, thunder, lightning, radar, car sparks, etc.).

To distinguish the solar radio signals from interference at millisecond scale is more difficult than at second-scale observations. The instruments and computer programs of observations on $1 \mathrm{~ms}$ time scale are still developing, and need normalizations. On the other hand, during the minimum years of solar activity, there are only a few microwave bursts.

Therefore, not until this year have the repeated observations at the same wavelength and the corresponding observations at different wavelengths been far from enough, especially since we have not yet obtained the results observed simultaneously with $1 \mathrm{~ms}$ time resolution at the same wavelength and in different places. One must take caution in making use of these data (8) for the purpose of theoretical studies.

We hope to increase the ability of anti-interference of the equipment and conduct spectral observations of solar radio bursts with high resolution. We suggest that the solar microwave bursts on $1 \mathrm{~ms}$ time scale be carried out in the maximum years (1990-1991) of the 22 nd solar cycle.

\section{ACKNOWLEDGMENTS}

Yan Yuanyi, Zhou Li and Zhang Weixue of Beijing Observatory, and Yao Deyi and Lu Xi or Peking University, attended the observations and experiments of $21 \mathrm{~cm}$ radiometer with $\mathrm{ms}$ scale, and thanks to Professor Xiao Zuo of Peking University for his kind assistance. 


\section{REFERENCES:}

(1) Slottje, C., 1978, Nature, 275, 520.

(2) Kaufmann, P., 1978, Solar Phys., 60, 367.

(3) Kaufmann, P., Strauss, F. M. Opher, R. and LaPorte, C., 1980, Astron. Astrophys., 87, 58-62.

(4) Ren-yang Zhao and Sheng-zhen Jin, 1982, Scientia Sinica, 26, 422.

(5) Luo Xianhan, Yan Yuanyi, Yao Deyi, Zhou Li, Zhang Weixue and Lu Xi, 1984, Publications of the Beijing Astronomical Observatory, 7, 101-105.

(6) Hu Hanming, Lu Songquan, Gong Yuanfang, Shi Shuobiao and Shang Qiongzhen, $\quad 1988$, Publications of Yunnan Observatory, No. 1, pp.3944.

(7) Ji Shuchen, Xie Ruixiang and Zhang Fan, 1988, Publications of Yunnan Observatory, №. 1, p.92.

(8) Zheng Leping and Liu Yuying, 1988, ACTA Astronomica Sinica, 29, 175180. 


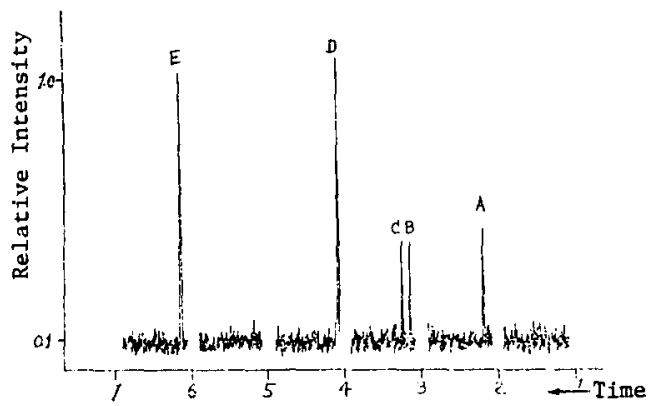

(S)

Fig. 1(a)

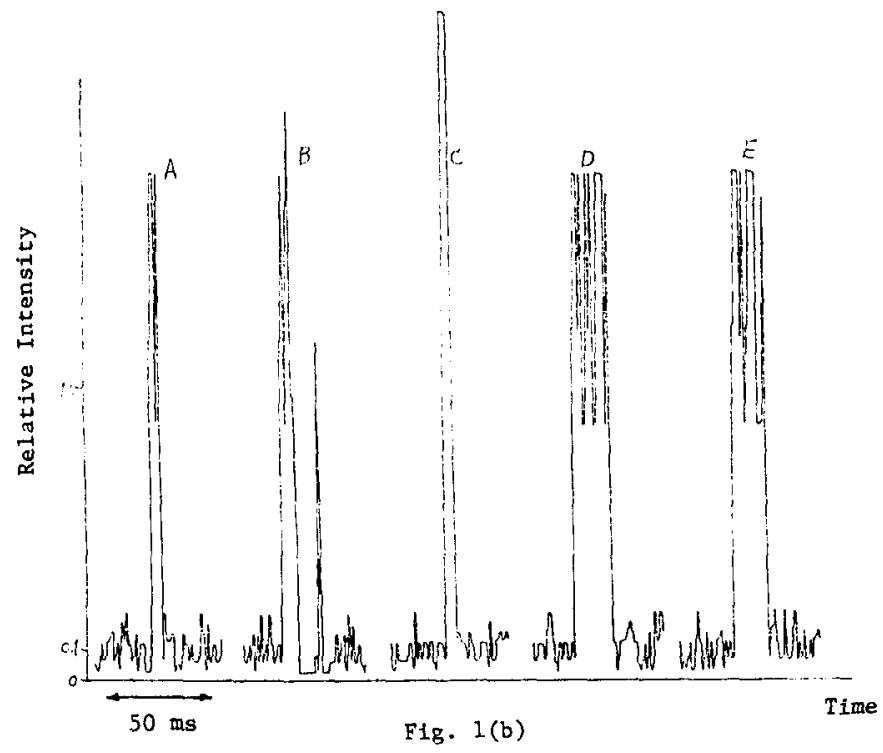



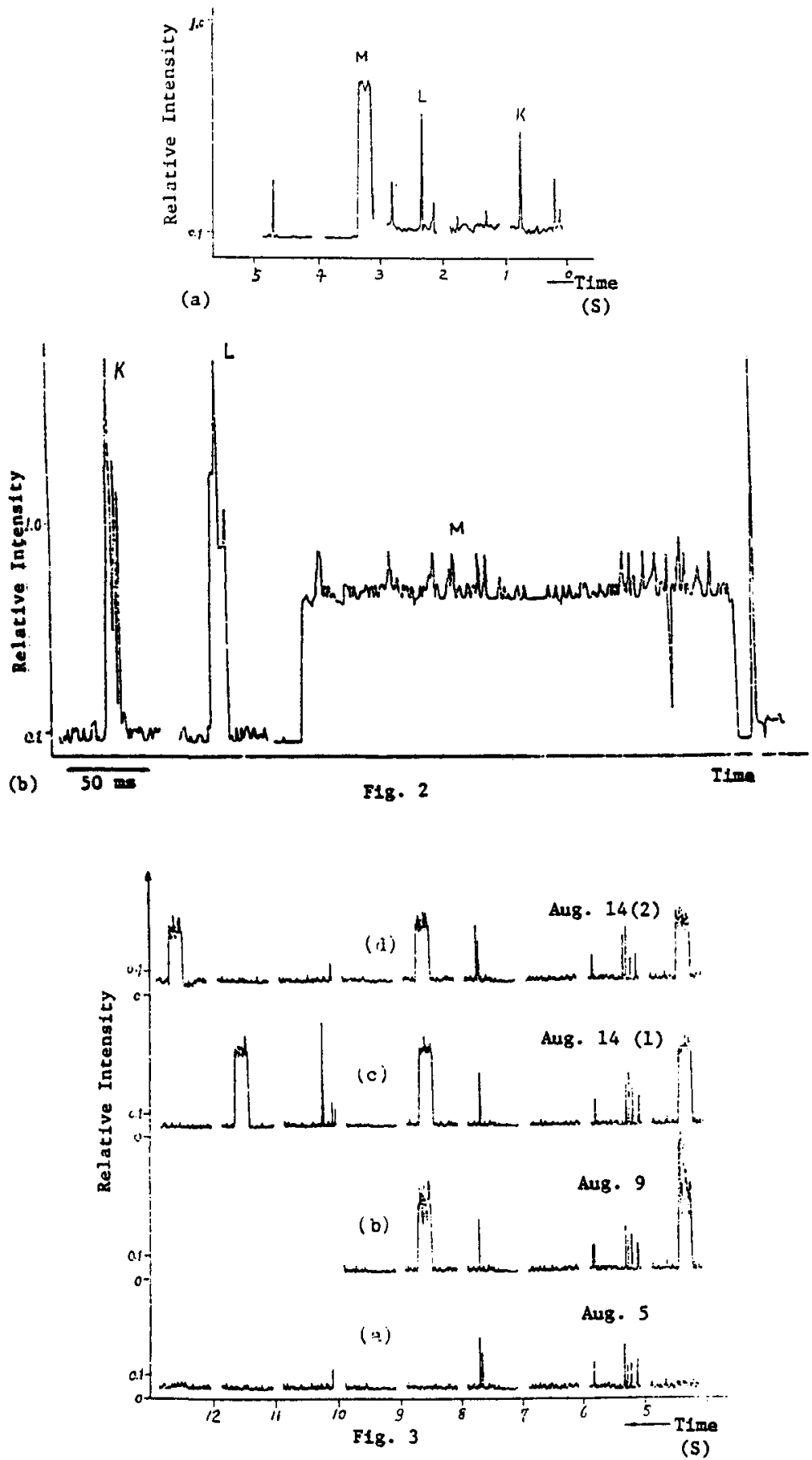